NBER WORKING PAPER SERIES

LIQUIDITY AND RISK MANAGEMENT

Nicolae B. Garleanu

Lasse H. Pedersen

Working Paper 12887

http://www.nber.org/papers/w12887

\author{
NATIONAL BUREAU OF ECONOMIC RESEARCH \\ 1050 Massachusetts Avenue \\ Cambridge, MA 02138 \\ February 2007
}

We are grateful for helpful conversations with Franklin Allen, Dimitri Vayanos, and Jeff Wurgler. The views expressed herein are those of the author(s) and do not necessarily reflect the views of the National Bureau of Economic Research.

(C) 2007 by Nicolae B. Garleanu and Lasse H. Pedersen. All rights reserved. Short sections of text, not to exceed two paragraphs, may be quoted without explicit permission provided that full credit, including $\odot$ notice, is given to the source. 
Liquidity and Risk Management

Nicolae B. Garleanu and Lasse H. Pedersen

NBER Working Paper No. 12887

February 2007

JEL No. G10

\begin{abstract}
$\underline{\text { ABSTRACT }}$
This paper provides a model of the interaction between risk-management practices and market liquidity. On one hand, tighter risk management reduces the maximum position an institution can take, thus the amount of liquidity it can offer to the market. On the other hand, risk managers can take into account that lower liquidity amplifies the effective risk of a position by lengthening the time it takes to sell it. The main result of the paper is that a feedback effect can arise: tighter risk management reduces liquidity, which in turn leads to tighter risk management, etc. This can help explain sudden drops in liquidity and, since liquidity is priced, in prices in connection with increased volatility or decreased risk-bearing capacity.
\end{abstract}

Nicolae B. Garleanu

University of Pennsylvania

Wharton School

3620 Locust Walk

2300 Steinberg-Dietrich Hall

Philadelphia, PA 19104-6137

and NBER

garleanu@wharton.upenn.edu

Lasse H. Pedersen

NYU Stern Finance

44 West Fouth Street

Suite 9-190

New York, NY 10012

and NBER

lpederse@stern.nyu.edu 


\section{Liquidity and Risk Management}

\section{Nicolae Gârleanu and Lasse Heje Pedersen}

This paper provides a model of the interaction between risk-management practices and market liquidity. Our main finding is that a feedback effect can arise: tighter risk management leads to market illiquidity, and this illiquidity further tightens risk management.

Risk management plays a central role in institutional investors' allocation of capital to trading. For instance, a risk manager may limit a trading desk's one-day $99 \%$ value at risk (VaR) to $\$ 1$ million. This means that the trading desk must choose a position such that, over the following day, its value drops no more than $\$ 1$ million with $99 \%$ probability. Risk management thus helps control an institution's use of capital while limiting default risk, and helps mitigate agency problems. Jorion (2000, page xxiii) states that VaR "is now increasingly used to allocate capital across traders, business units, products, and even to the whole institution."

We do not focus on the benefits of risk management within an institution adopting such controls, but, rather, on the aggregate effects of such practices on liquidity and asset prices. An institution may benefit from tightening its risk management and restricting its security position, but as a consequence it cannot provide as much liquidity to others. We show that, if everyone uses a tight risk management, then market liquidity is lowered in that it takes longer to find a buyer with unused risk-bearing capacity, and, since liquidity is priced, prices 
fall.

Not only does risk management affect liquidity, but liquidity can also affect risk-management practices. For instance, Bank for International Settlements (2001, page 15) states that "For the internal risk management, a number of institutions are exploring the use of liquidity adjusted-VaR, in which the holding periods in the risk assessment are adjusted to account for market liquidity, in particular by the length of time required to unwind positions." For instance, if liquidation is expected to take two days, a two-day VaR might be used instead of a one-day VaR. Since a security's risk over two days is greater than over one day, this means that a trader must choose a smaller position to satisfy his liquidity-adjusted value at risk (LVaR) constraint. One motivation for this constraint is that, if an institution needs to sell, its maximum loss before the completion of the sale is limited by the LVaR.

The main result of the paper is that subjecting traders to an LVaR gives rise to a multiplier effect: tighter risk management leads to more restricted positions, hence longer expected selling times, implying higher risk over the expected selling period, which further tightens the risk management, and so on. This snowballing feedback between liquidity and risk management can help explain why liquidity can suddenly drop. We show that this spiraling illiquidity can arise if volatility rises, or if more agents face reduced risk-bearing capacity for instance, because of investor redemptions, losses, or increased risk aversion.

Our link between liquidity and risk management is a testable prediction. While no formal empirical evidence is available, to our knowledge, our prediction is consistent with anecdotal evidence on financial market crises. For example, in August 1998 several traders lost money due to a default of Russian bonds and, simultaneously, market volatility increased. As a 
result, the (L)VaR of many investment banks and other institutions increased. To bring risk back in line, many investment banks reportedly asked traders to reduce positions, leading to falling prices and lower liquidity. These market moves exacerbated the risk-management problems, fueling the crisis in a similar manner to the one modelled here.

We capture these effects by extending the search model for financial securities of Duffie, Gârleanu, and Pedersen (2005, 2007) (DGP). This framework of time-consuming search is well suited for modeling liquidity-based risk management as it provides a natural framework for studying endogenous selling times. While DGP relied on exogenous position limits, we endogenize positions based on a risk-management constraint, and consider both a simple and a liquidity-adjusted VaR. Hence, we solve the fixed-point problem of jointly calculating endogenous positions given the risk-management constraint and computing the equilibrium (L) VaR given the endogenous positions that determine selling times and price volatility. Weill (2007) considers another extension of DGP in which marketmaker liquidity provision is limited by capital constraints. Our multiplier effect is similar to that of Brunnermeier and Pedersen (2006) who show that liquidity and traders' margin requirements can be mutually reinforcing.

\section{$1 \quad$ Model}

The economy has two securities: a "liquid" security with risk-free return $r$ (i.e. a "moneymarket account"), and a risky illiquid security. The risky security has a dividend-rate process $X$ and a price $P(X)$, which is determined in equilibrium. The dividend rate is Lévy with 
finite variance. It has a constant drift normalized to zero, $\mathrm{E}_{t}(X(t+T)-X(t))=0$, and a volatility $\sigma_{X}>0$, i.e.,

$$
\operatorname{Var}_{t}(X(t+T)-X(t))=\sigma_{X}^{2} T \text {. }
$$

Examples include Brownian motions, Poisson processes, and sums of these.

The economy is populated by a continuum of agents who are risk neutral and infinitely lived, have a time-preference rate equal to the risk-free interest rate $r>0$, and must keep their wealth bounded from below. Each agent is characterized by an intrinsic type $i \in\{h, l\}$, which is a Markov chain, independent across agents, and switching from $l$ ("low") to $h$ ("high") with intensity $\lambda_{u}$, and back with intensity $\lambda_{d}$. An agent of type $i$ holding $\theta_{t}$ shares of the asset incurs a holding cost of $\delta>0$ per share and per unit of time if he violates his risk-management constraint

$$
\operatorname{var}_{t}\left(\theta_{t}\left[P\left(X_{t+\tau}\right)-P\left(X_{t}\right)\right]\right) \leq\left(\sigma^{i}\right)^{2}
$$

where $\sigma^{i}$ is the risk-bearing capacity, defined by $\sigma^{h}=\bar{\sigma}>0$ and $\sigma^{l}=0$. The low risk-bearing capacity of the low-type agents can be interpreted as a need for more stable earnings, hedging reasons to reduce a position, high financing costs, or a need for cash (e.g., an asset manager whose investors redeem capital). ${ }^{1}$

We use this constraint as a parsimonious way of capturing risk constraints, such as the

\footnotetext{
${ }^{1}$ An interesting extension of our model would consider the direct benefit of tighter risk management, which could be captured by a lower $\lambda_{d}$.
} 
very popular VaR constraint, ${ }^{2}$ which are used by most financial institutions. Our results are robust in that they only rely on two natural properties of the measure of risk: the risk measure increases with the size of the security position and the length of the time period $\tau$ over which the risk is assessed. While the constraint is not endogenized in the model, we note that its wide use in the financial world is probably due to agency problems, default risk, and the need to allocate scarce capital.

We consider two types of risk management: (a) "simple risk management," in which the variance of the position in (2) is computed over a fixed time horizon $\tau$, and (b) "liquidityadjusted risk management," in which the variance is computed over the time required for selling the asset to an unconstrained buyer, which will be a random equilibrium quantity.

Because agents are risk neutral and we are interested in a steady-state equilibrium, we restrict attention to equilibria in which, at any given time and state of the world, an agent holds either 0 or $\bar{\theta}$ units of the asset, where $\bar{\theta}$ is the largest position that satisfies (2) with $\sigma^{i}=\bar{\sigma}$, taking the prices and search times as given. ${ }^{3}$ Hence, the set of agent types is $\mathcal{T}=\{h o, h n, l o, \ln \}$, with the letters " $h$ " and "l" designating the agent's current intrinsic risk-bearing state as high or low, respectively, and with " $o$ " or " $n$ " indicating whether the agent currently owns $\bar{\theta}$ shares or none, respectively. We let $\mu_{\zeta}(t)$ denote the fraction at time

\footnotetext{
${ }^{2} \mathrm{~A}$ VaR constraint stipulates that $\operatorname{Pr}\left(-\theta\left[P\left(X_{t+\tau}\right)-P\left(X_{t}\right)\right] \geq V a R\right) \leq \pi$ for some risk limit VaR and some confidence level $\pi$. If $X$ is a Brownian motion, this is the same as (2). We note that rather than considering only price risk, we could alternatively consider the risk of the gains process (i.e., including dividend risk) $G_{t, \tau}=P(X(t+\tau))-P(X(t))+\int_{t}^{t+\tau} X(s) d s$. This yields qualitatively similar results (and quantitatively similar for many reasonable parameters since dividend risk is orders of magnitude smaller than price risk over a small time period).

${ }^{3}$ Note that the existence of such an equilibrium requires that the risk limit $\bar{\sigma}$ be not too small relative to the total supply $\Theta$, a condition that we assume throughout.
} 
$t$ of agents of type $\zeta \in \mathcal{T}$. These fractions add up to 1 and markets must clear:

$$
\begin{aligned}
1 & =\mu_{h o}+\mu_{h n}+\mu_{l o}+\mu_{l n} \\
\Theta & =\bar{\theta}\left(\mu_{h o}+\mu_{l o}\right)
\end{aligned}
$$

where $\Theta>0$ is the total supply of shares per investor.

Central to our analysis is the notion that the risky security is not perfectly liquid, in the sense that an agent can only trade it when she finds a counterparty. Every agent finds a potential counterparty, selected randomly from the set of all agents, with intensity $\lambda$, where $\lambda \geq 0$ is an exogenous parameter characterizing the market liquidity for the asset. Hence, the intensity of finding a type- $\zeta$ investor is $\lambda \mu_{\zeta}$, that is, the search intensity multiplied by the fraction of investors of that type. When two agents meet, they bargain over the price, with the seller having bargaining power $q \in[0,1]$.

This model of illiquidity captures directly the search that characterizes over-the-counter (OTC) markets. In these markets, traders must find an appropriate counterparty, which can be time consuming. Trading delays also arise due to time spent gathering information, reaching trading decisions, mobilizing capital, etc. Hence, trading delays are commonplace and, therefore, the model can also capture features of other markets such as specialist and electronic limit-order-book markets, although these markets are, of course, distinct from OTC markets. 


\section{Equilibrium Risk Management, Liquidity, and Prices}

We now proceed to derive the steady-state equilibrium agent fractions $\mu$, the maximum holding $\bar{\theta}$, and the price $P$. Naturally, low-type owners $l o$ want to sell and high-type nonowners $h n$ want to buy, which leads to

$$
0=-2 \lambda \mu_{h n}(t) \mu_{l o}(t)-\lambda_{u} \mu_{l o}(t)+\lambda_{d} \mu_{h o}(t)
$$

and three more such steady-state equations. Equation (5) states that the change in the fraction of lo agents has three components, corresponding to the three terms on the right hand side: First, whenever an lo agent meets an $h n$ investor, he sells his asset and is no longer an lo agent. Second, whenever the intrinsic type of a lo agent switches to high, he becomes an ho agent. Third, ho agents can switch type and become lo. Duffie, Gârleanu, and Pedersen (2005) show that, taking $\bar{\theta}$ as fixed, there is a unique stable steady-state mass

distribution as long as $\bar{\theta} \geq \Theta$. Here, agents' positions $\bar{\theta}$ are endogenous and depend on $\mu$, so that we must calculate a fixed point.

Agents take the steady-state distribution $\mu$ as fixed when they derive their optimal strategies and utilities for remaining lifetime consumption, as well as the bargained price $P$. The utility of an agent depends on his current type, $\zeta(t) \in \mathcal{T}$, (i.e., whether he is a high or a low type and whether he owns zero or $\bar{\theta}$ shares), the current dividend $X(t)$, and the wealth $W(t)$ in his bank account:

$$
V_{\zeta}\left(X(t), W_{t}\right)=W_{t}+1_{(\zeta \in\{h o, l o\})} \frac{\bar{\theta} X(t)}{r}+\bar{\theta} v_{\zeta},
$$


where the type-dependent utility coefficients $v_{\zeta}$ are to be determined. With $q$ the bargaining power of the seller, bilateral Nash bargaining yields the price:

$$
P \bar{\theta}=\left(V_{l o}-V_{l n}\right)(1-q)+\left(V_{h o}-V_{h n}\right) q .
$$

We conjecture, and later confirm, that the equilibrium asset price per share is of the form

$$
P(X(t))=\frac{X(t)}{r}+p
$$

for a constant $p$ to be determined. The value-function coefficients $v_{\zeta}$ and $p$ are given by a set of Hamilton-Jacobi-Bellman equations, stated and solved in the appendix. The appendix contains all the proofs.

Proposition 1 If the risk-limit $\bar{\sigma}$ is sufficiently large, there exists an equilibrium with holdings 0 and $\bar{\theta}$ that satisfy the risk management constraint (2) with equality for low- and high-type agents, respectively. With simple risk management, the equilibrium is unique and

$$
\bar{\theta}=\frac{r \bar{\sigma}}{\sigma_{X}} \frac{1}{\sqrt{\tau}}
$$

With liquidity-adjusted risk management, $\bar{\theta}$ depends on the equilibrium fraction of potential buyers $\mu_{h n}$ and satisfies

$$
\bar{\theta}=\frac{r \bar{\sigma}}{\sigma_{X}} \sqrt{2 \lambda \mu_{h n}}
$$


In both cases, the equilibrium price is given by

$$
P\left(X_{t}\right)=\frac{X_{t}}{r}-\frac{\delta}{r} \frac{r(1-q)+\lambda_{d}+2 \lambda \mu_{l o}(1-q)}{r+\lambda_{d}+2 \lambda \mu_{l o}(1-q)+\lambda_{u}+2 \lambda \mu_{h n} q},
$$

where the fractions of agents $\mu$ depend on the type of risk management.

These results are intuitive. The "position limit" $\bar{\theta}$ increases in the risk limit $\bar{\sigma}$ and decreases in the asset volatility and in the square root of the VaR period length, which is $\tau$ under simple risk management and $\left(2 \lambda \mu_{h n}\right)^{-1}$ under liquidity-adjusted risk management. In the latter case, position limits increase in the search intensity $\lambda$ and in the fraction of eligible buyers $\mu_{h n}$.

The price equals the present value of dividends, $\frac{X_{t}}{r}$, minus a discount for illiquidity. Naturally, the liquidity discount is larger if there are more low-type owners in equilibrium ( $\mu_{l o}$ is larger) and fewer high-type non-owners ready to buy ( $\mu_{h n}$ is smaller).

Of the equilibria obtaining with liquidity-adjusted risk management, we concentrate on the ones that are stable, in the sense that increasing $\bar{\theta}$ marginally would result in equilibrium quantities violating the VaR constraint (2). Conversely, an equilibrium is unstable if a marginal change in holdings that violates the constraint would result in the equilibrium adjusting so that the constraint is not violated. If an equilibrium exists, then a stable equilibrium exists. Indeed, the equilibrium with the largest $\bar{\theta}$ is stable and has the highest welfare among all equilibria.

The main result of the paper characterizes the equilibrium connection between liquidity and risk management: 

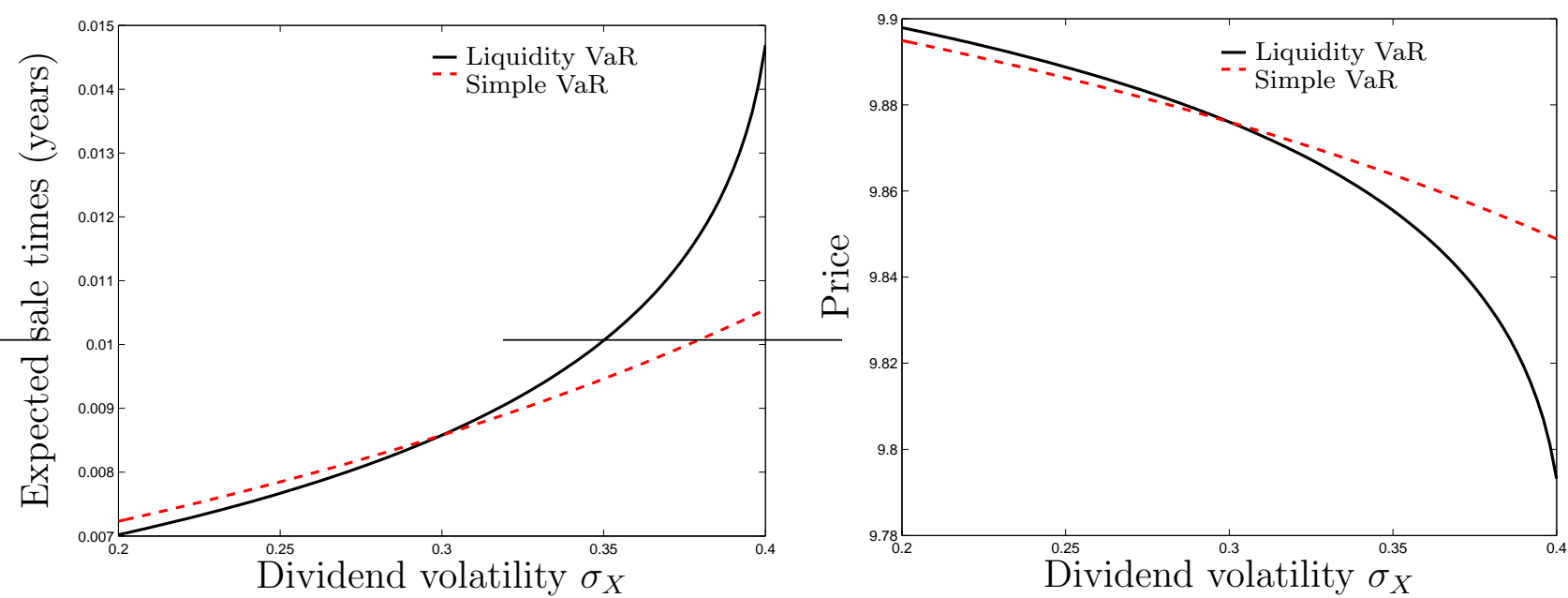

Figure 1: The effects of dividend volatility on equilibrium seller search times (left panel) and prices (right panel) with, respectively simple (dashed line) and liquidity-adjusted (solid line) risk management.

Proposition 2 Suppose that $\bar{\sigma}$ is large enough for existence of an equilibrium. Consider a stable equilibrium with liquidity-adjusted risk management and let $\tau=\frac{1}{2 \lambda \mu_{h n}}$, which means that the equilibrium allocations and price are the same with simple risk management.

Consider any combination of the conditions (a) higher dividend volatility $\sigma_{X}$, (b) lower risk limit $\bar{\sigma},(c)$ lower meeting intensity $\lambda,(d)$ lower switching intensity $\lambda_{u}$ to the high riskbearing state, and (e) higher switching intensity $\lambda_{d}$ to the low risk-bearing state. Then, (i) the equilibrium position $\bar{\theta}$ decreases, (ii) expected search times for selling increase, and (iii) prices decrease. All three effects are larger with liquidity-adjusted risk management.

To see the intuition for these results, consider first the impact of a higher dividend volatility. This makes the risk-management constraint tighter, inducing agents to reduce their positions and spreading securities among more agents, thus leaving a smaller fraction of agents with unused risk-bearing capacity. Hence, sellers' search time increase and their 
bargaining position worsen, leading to lower prices. This price drop is due to illiquidity, as agents are risk neutral. ${ }^{4}$

Importantly, with liquidity-adjusted risk management, the increased search time for sellers means that the risk over the expected liquidation period rises, thus further tightening the risk-management constraint, further reducing positions, further increasing search times, and so on.

This multiplier also increases the sensitivity of the economy with liquidity-adjusted risk management to the other shocks (b)-(e). Indeed, a lower risk limit (b) is equivalent to a higher dividend risk. The "liquidity shocks" (c)-(e) do not affect the equilibrium position $\bar{\theta}$ with simple risk management, but they do increase the sellers' search times and reduce prices. With liquidity-adjusted risk management, these liquidity shocks reduce security positions, too, because of increased search times and, as explained above, a multiplier effect arises.

The multiplier arising from the feedback between trading liquidity and risk management clearly magnifies the effects on liquidity and prices of changes in the economic environment. Our steady-state model illustrate this point using comparative static analysis that essentially compares across economies. Similar results would arise in the time series of a single economy if there was random variation in the model characteristic, e.g., parameters switched in a Markov chain as in Duffie, Gârleanu, and Pedersen (2007). In the context of such time-series variation, our multiplier effect can generate the abrupt changes in prices and selling times that characterize crises.

\footnotetext{
${ }^{4}$ In a Walrasian market with immediate trade, the price is the present value of dividends $\frac{X}{r}$ when $\frac{\Theta}{\theta}<$ $\frac{\lambda_{u}}{\lambda_{u}+\lambda_{d}}$, a condition that is satisfied is all our examples. (When $\frac{\Theta}{\theta}>\frac{\lambda_{u}}{\lambda_{u}+\lambda_{d}}$, the Walrasian price is $\frac{X-\delta}{r}$.)
} 
We illustrate our model with a numerical example in which $\lambda=100, r=10 \%, X_{0}=1$, $\lambda_{d}=0.2, \lambda_{u}=2, \delta=3, q=0.5, \Theta=1$, and $\bar{\sigma}=1$. Figure 1 shows how prices (right panel) and sellers' expected search times (left panel) depend on asset volatility. The solid line shows this for liquidity-adjusted risk management and the dashed for simple risk management with $\tau=0.0086$, which is chosen so that the risk management schemes are identical for $\sigma_{X}=30 \%$. Search times increase and prices decrease with volatility. Importantly, these sensitivities are stronger (i.e., the curves are steeper) with liquidity-adjusted risk management due to the interaction between market liquidity (i.e., search times) and risk management.

\section{References}

Brunnermeier, M. and L. H. Pedersen (2006). Market Liquidity and Funding Liquidity. Working paper, Princeton University.

Duffie, D., N. Gârleanu, and L. H. Pedersen (2005). Over-the-Counter Markets. Econometrica 73, 1815-1847.

Duffie, D., N. Gârleanu, and L. H. Pedersen (2007). Valuation in Over-the-Counter Markets. Review of Financial Studies, forthcoming.

Joint Forum (2001). Multidisciplinary Working Group on Enhanced Disclosure. Bank for International Settlements.

Jorion, P. (2000). Value at Risk, Second Edition. New York: McGraw-Hill.

Weill, P.-O. (2007). Leaning Against the Wind. Review of Economic Studies, forthcoming. 


\section{A Appendix}

\section{Steady-State Distribution of Agent Types:}

The steady-state distribution of agent types is given by the following system of equations.

The economic intuition for these equations is discussed in connection with Equation (5) in the paper.

$$
\begin{gathered}
0=\dot{\mu}_{l o}(t)=-2 \lambda \mu_{h n}(t) \mu_{l o}(t)-\lambda_{u} \mu_{l o}(t)+\lambda_{d} \mu_{h o}(t) \\
0=\dot{\mu}_{h n}(t)=-2 \lambda \mu_{h n}(t) \mu_{l o}(t)-\lambda_{d} \mu_{h n}(t)+\lambda_{u} \mu_{l n}(t) \\
0=\dot{\mu}_{h o}(t)=2 \lambda \mu_{h n}(t) \mu_{l o}(t)-\lambda_{d} \mu_{h o}(t)+\lambda_{u} \mu_{l o}(t) \\
0=\dot{\mu}_{l n}(t)=2 \lambda \mu_{h n}(t) \mu_{l o}(t)-\lambda_{u} \mu_{l n}(t)+\lambda_{d} \mu_{h n}(t) .
\end{gathered}
$$

Two of the equations in (A.1) are redundant, so that, together with (3)-(4), (A.1) forms a well-posed system. The system can be reduced to the quadratic equation

$$
0=2 \lambda \mu_{h n}^{2}+\left(2 \lambda\left(\frac{\Theta}{\bar{\theta}}-\frac{\lambda_{u}}{\lambda_{d}+\lambda_{u}}\right)+\lambda_{u}+\lambda_{d}\right) \mu_{h n}-\lambda_{u}\left(1-\frac{\Theta}{\bar{\theta}}\right)
$$

We use below the following result, which follows from calculations in Duffie, Gârleanu, and Pedersen (2007).

Lemma 3 If $\bar{\theta} \geq \Theta$, the system of equations (3), (4), and (A.1) has a unique solution in $[0,1]^{4}$. The steady-state fraction of sellers $\mu_{l o}$ increases with $\lambda_{d}$ and decreases with $\lambda_{u}$, while the fraction of buyers $\mu_{h n}$ decreases with $\lambda_{d}$ and increases with $\lambda_{u}$. Both $\mu_{l o}$ and $\mu_{h n}$ decrease with the meeting intensity $\lambda$. Furthermore, $\mu_{l o}$ increases, while $\mu_{\text {hn }}$ decreases with $\bar{\theta}$. 


\section{Proof of Proposition 1:}

The value function coefficients are given by

$$
\begin{aligned}
0 & =r v_{l o}-\lambda_{u}\left(v_{h o}-v_{l o}\right)-2 \lambda \mu_{h n}\left(p-v_{l o}+v_{l n}\right)+\delta \\
0 & =r v_{l n}-\lambda_{u}\left(v_{h n}-v_{l n}\right) \\
0 & =r v_{h o}-\lambda_{d}\left(v_{l o}-v_{h o}\right) \\
0 & =r v_{h n}-\lambda_{d}\left(v_{l n}-v_{h n}\right)-2 \lambda \mu_{h o}\left(v_{h o}-v_{h n}-p\right) \\
p & =\left(v_{l o}-v_{l n}\right)(1-q)+\left(v_{h o}-v_{h n}\right) q .
\end{aligned}
$$

The first equation means that an agent of type lo has a zero change in steady-state utility.

The change in his utility is due to opportunity cost $-r v_{l o}$, expected change in intrinsic-type $\lambda_{u}\left(v_{h o}-v_{l o}\right)$, trade $2 \lambda \mu_{h n}\left(p-v_{l o}+v_{l n}\right)$, and holding cost $-\delta$. The next three equations are similar. Direct solution of this system yields

$$
p=\frac{\delta}{r} \frac{r(1-q)+\lambda_{d}+2 \lambda \mu_{l o}(1-q)}{r+\lambda_{d}+2 \lambda \mu_{l o}(1-q)+\lambda_{u}+2 \lambda \mu_{h n} q} .
$$

Given the dependence of $P\left(X_{t}\right)$ on $X_{t}$, it is immediate that

$$
\operatorname{var}_{t}\left(P\left(X_{t+\tau}\right)-P\left(X_{t}\right)\right)=\frac{\sigma_{X}^{2}}{r^{2}} \tau
$$


for constant $\tau$. If $\tau$ is randomly distributed with constant arrival intensity $\lambda \mu_{h n}$,

$$
\begin{aligned}
\operatorname{var}_{t}\left(P\left(X_{t+\tau}\right)-P\left(X_{t}\right)\right) & =\frac{1}{r^{2}} \operatorname{var}_{t}\left(X_{t+\tau}-X_{t}\right) \\
& =\frac{1}{r^{2}}\left[E_{t}\left(\operatorname{var}_{t}\left(X_{t+\tau}-X_{t} \mid \tau\right)\right)+\operatorname{var}_{t}\left(E_{t}\left(X_{t+\tau}-X_{t} \mid \tau\right)\right)\right] \\
& =\frac{1}{r^{2}}\left[\sigma_{X}^{2} E_{t}(\tau)\right]=\frac{\sigma_{X}^{2}}{r^{2} \lambda \mu_{h n}},
\end{aligned}
$$

and it is clear that, when the VaR constraint (2) binds, the equilibrium holding $\theta$ is given by (9) or (10), depending on the nature of risk management.

Proof of Proposition 2: The equilibrium with the two kinds of risk management is given by $f_{i}(\bar{\theta})=\frac{r \bar{\sigma}}{\sigma_{X}}$, where $f_{0}(\bar{\theta})=\bar{\theta} \sqrt{\tau}$ and $f_{1}(\bar{\theta})=\frac{\bar{\theta}}{\sqrt{2 \lambda \mu_{h n}(\bar{\theta})}}$. Clearly, $f_{0}=f_{1}$ when $\tau=\frac{1}{2 \lambda \mu_{h n}}$, so that the two equilibria are identical.

The sensitivity $\bar{\theta}^{\prime}$ of the equilibrium position $\bar{\theta}$ to the ratio $\frac{\bar{\sigma}}{\sigma_{X}}$ is given by $f_{i}^{\prime} \bar{\theta}^{\prime}=r$. With simple risk management, it is clear that $f_{0}^{\prime}>0$, so that the equilibrium position $\bar{\theta}$ decreases if the volatility $\sigma_{X}$ increases or the risk limit $\bar{\sigma}$ decreases. A decreasing $\bar{\theta}$ leads, in turn, to an increasing expected search time for sellers $\left(2 \lambda \mu_{h n}\right)^{-1}$ and a decreasing price, because $\partial \mu_{h n} / \partial \bar{\theta}>0$ and $\partial \mu_{l o} / \partial \bar{\theta}<0$, as stated by Lemma 3 .

With liquidity-adjusted risk management, $f_{1}^{\prime}>0$ by the definition of a stable equilibrium, and, since $\partial \mu_{h n} / \partial \bar{\theta}>0, f_{1}^{\prime}<f_{0}^{\prime}$. Hence, with liquidity-adjusted risk management, the effects of $\sigma_{X}$ on the equilibrium quantities are larger in absolute value and of the same sign as with simple risk management. A stable equilibrium exists because $f_{1}<\infty$ on $(\Theta, \infty)$, while $\lim _{x \rightarrow \Theta} f_{1}(x)=\lim _{x \rightarrow \infty} f_{1}(x)=\infty$, given that $\mu_{h n}(\Theta)=0$ and $\lim _{x \rightarrow \infty} \mu_{h n}(x)>0$. 
Consider now the dependence on the meeting intensity $\lambda$. It holds that

$$
\begin{aligned}
0 & =\frac{d f_{i}}{d \lambda} \\
& =\frac{\partial f_{i}}{\partial \bar{\theta}} \frac{d \bar{\theta}}{d \lambda}+\frac{\partial f_{i}}{\partial \lambda}+\frac{\partial f_{i}}{\partial \mu_{h n}} \frac{d \mu_{h n}}{d \lambda} \\
& =\frac{\partial f_{i}}{\partial \bar{\theta}} \frac{d \bar{\theta}}{d \lambda}+\frac{\partial f_{i}}{\partial \lambda}+\frac{\partial f_{i}}{\partial \mu_{h n}}\left(\frac{\partial \mu_{h n}}{\partial \lambda}+\frac{\partial \mu_{h n}}{\partial \bar{\theta}} \frac{d \bar{\theta}}{d \lambda}\right)
\end{aligned}
$$

which can be solved for $\frac{d \bar{\theta}}{d \lambda}$.

With simple risk management, it follows that $\frac{d \bar{\theta}}{d \lambda}=0$, as $\frac{\partial f_{0}}{\partial \lambda}=\frac{\partial f_{0}}{\partial \mu_{h n}}=0$. With liquidityadjusted risk management, $\frac{d \bar{\theta}}{d \lambda}>0$, since $\frac{\partial f_{1}}{\partial \bar{\theta}}+\frac{\partial f_{1}}{\partial \mu_{h n}} \frac{\partial \mu_{h n}}{\partial \bar{\theta}}=\frac{d f_{1}}{d \bar{\theta}}>0$ for a stable equilibrium. We also use the fact that $\frac{\partial f_{1}}{\partial \lambda}+\frac{\partial f_{1}}{\partial \mu_{h n}} \frac{\partial \mu_{h n}}{\partial \lambda}<0$, which holds because $\frac{d\left(\lambda \mu_{h n}\right)}{d \lambda}>0$, as can be shown based on (A.2). Since $\frac{\partial f_{1}}{\partial \lambda}<0$, the result on selling times also obtains.

The price effects follows from

$$
\frac{d P}{d \lambda}=\frac{\partial P}{\partial \lambda}+\frac{\partial P}{\partial \bar{\theta}} \frac{d \bar{\theta}}{d \lambda}
$$

The first term gives the impact with simple risk management, while the second captures the additional impact introduced by adjusting risk management to liquidity. Since $\frac{\partial P}{\partial \theta}>0$ from Proposition 1 and (A.2) (a complete proof can be given along the lines of Duffie, Gârleanu, and Pedersen (2007)), the second term has the same sign as $\frac{d \bar{\theta}}{d \lambda}$. This sign was shown above to be positive, as is the sign of $\frac{\partial P}{\partial \lambda}$. The total effect is therefore larger with risk-adjusted risk management.

Similar reasoning establishes the results concerning the dependence on $\lambda_{d}$ and $\lambda_{u}$. 